\title{
ANSÄTZE VON NACHHALTIGKEIT IM UMGANG MIT HISTORISCHEN GÄRTEN
}

\begin{abstract}
Die Bewirtschaftung, Pflege und Unterhaltung von historischen Gärten muss gerade in Zeiten eines sich wandelnden Klimas nachhaltig gestaltet werden. Dazu gehören die in diesem Beitrag dargestellten Aspekte einer Wiederanlage und Bewirtschaftung von Nutzgartenpartien, der Betrieb von Parkbaumschulen und ein Pflegemanagement, das geschlossene Stoffkreisläufe zum Ziel hat. Alle drei Ansätze haben Vorbilder im historisch dokumentierten Umgang mit Parks und Gärten im 18. und 19. Jahrhundert.
\end{abstract}

The management, care and maintenance of historic gardens must be undertaken in a sustainable manner particularly during periods of climate change. This includes the aspects covered in this article relating to the reconstruction and management of kitchen garden sections, the running of park tree nurseries and care management that aims to establish closed material cycles. All three approaches can draw on exemplary models in the historically documented management of parks and gardens in the $18^{\text {th }}$ and $19^{\text {th }}$ centuries.

In der Gartendenkmalpflege geht es um die Bewahrung von wertvollen historischen Grünanlagen, die als kulturelles Erbe verstanden werden. Diese Bewahrung hat bei dem aus lebendigem Baumaterial geschaffenen System Park oder Garten immer mit Pflege, im gartendenkmalpflegerischen Fachbegriff mit Instandhaltung, zu tun. Grundsätzlich ist die Instandhaltung darauf ausgerichtet, die Gärten dauerhaft in ihrer Eigenschaft als Geschichtszeugnis und Kunstwerk zu erhalten - auch bei sich ändernden äußeren Rahmenbedingungen wie beispielsweise in Zeiten des Klimawandels. Ein dauerhafter Erhalt, der ein sich wandelndes System betrifft, muss nachhaltig gestaltet sein. Ansätze einer solchen nachhaltigen Parkpflege werden im Folgenden dargestellt.

Im Text werden Maskulinum und Femininum verwendet, wenn es um Personen geht. Gemeint sind grundsätzlich alle Menschen, gleich welcher Geschlechtsidentität sie sich zugehörig fühlen. 
Unter den verschiedenen Definitionen von Nachhaltigkeit soll hier diejenige verwendet werden, die Nachhaltigkeit als ein Handlungsprinzip zur Ressourcen-Nutzung versteht, das der Bewahrung der wesentlichen Eigenschaften, der Stabilität und der natürlichen Regenerationsfähigkeit des jeweiligen Systems dient. Wenn das System ein historischer Garten ist, geht es also darum, diesen als ganzheitliches System zu bewirtschaften, um ihn in seinen spezifischen Eigenarten dauerhaft tradieren zu können. Drei Aspekte möchte ich dabei hervorheben: Zum einen die Integration bzw. Re-Integration von Nutzpflanzen in historische Parks, die als Versorgungsgrundlage für die Gartenbesitzer bis ins 20. Jahrhundert eine zentrale Rolle gespielt haben. Des Weiteren ist die Eigenproduktion der Gehölze in Parkbaumschulen ein Aspekt, der die Resilienz des wichtigsten Bau-Materials und damit des gesamten Gartens erhöht. Ein ebenso grundlegender Ansatz ist das Schließen von Stoffkreisläufen bei der Parkpflege, um z.B. durch Kompost- und Mulchwirtschaft `Grünabfall in fruchtbares `Grüngut $`$ zu verwandeln und so die Standortqualitäten historischer Gärten langfristig zu sichern.

\section{Re-Integration von Nutzpflanzen in historischen Parks \\ Historische Situation in den Parkanlagen bis zum 19. Jahrhundert}

Quartiere für Obst und Gemüse waren immer ein Teil der Gärten. Die Verbindung von Schönheit und Nutzen ist in den gartentheoretischen Schriften seit der Antike belegt. Schon Varro beschreibt im ersten vorchristlichen Jahrhundert die Rasterpflanzung von Obstbäumen in der Quincunx-Anordnung als ein Beispiel für etwas, »was beim Anblick lieblicher erscheint, folgerichtig auch von größerem Ertrage ist « (Buch I, Kap. 7, 37 v. Chr., zit. n. Wimmer 1989, 1). Petrus Crescentius erwähnt in der Ruralia Commodora am Anfang des 14. Jahrhunderts Gärten aus Obstbaumpflanzungen und Reben, »die Vergnügen und Nutzen gewähren« (zit. n. Wimmer 1989, 26), und Johann Peschel beschreibt in seiner Gartenordnung von 1597 Gartenwege, die von zierenden Spalieren begleitet werden. Diese Spaliere könnten mit Johannisbeeren, Rosen, Berberitzen oder Stachelbeeren besetzt sein und auch zu überwölbten Gängen geschlossen werden, die dann mit Kürbissen, Bohnen oder Wein berankt werden. In dieser Form seien sie eine »zier und wolstandt in einem Garten und bringen neben der zier nicht geringen nutzen « (zit. n. Wimmer 1989, 72). Zur gleichen Zeit veröffentlicht Olivier de Serres in Paris das Werk Le Théatre d'Agriculture, in dem er auch über Gärten spricht. Küchengärten empfiehlt er in einem Längen-Breitenverhältnis von 5:3 anzulegen und durch Alleen zu gliedern, um »so dem Garten hinsichtlich der Vielfalt mehr Anmut [zu] verleihen « (zit. n. Wimmer 1989, 80). In seinen Beispielentwürfen für Küchengärten zeigt er Möglichkeiten auf, wie ein solcher Raum mit ästhetischen Qualitäten gestaltet werden kann (Abb. 1). Beete, die normalerweise in längsrechteckiger Form angelegt werden, könnten auch »zum Vergnügen« in drei-, fünf-, sechs-, sieben- und achteckigen Figuren gestaltet und »als Dekoration « mit ausdauernden Kräutern wie Lavendel, Wermut, Rosmarin, Petersilie oder Ysop eingefasst werden (zit. n. Wimmer 1989, 80). Im 


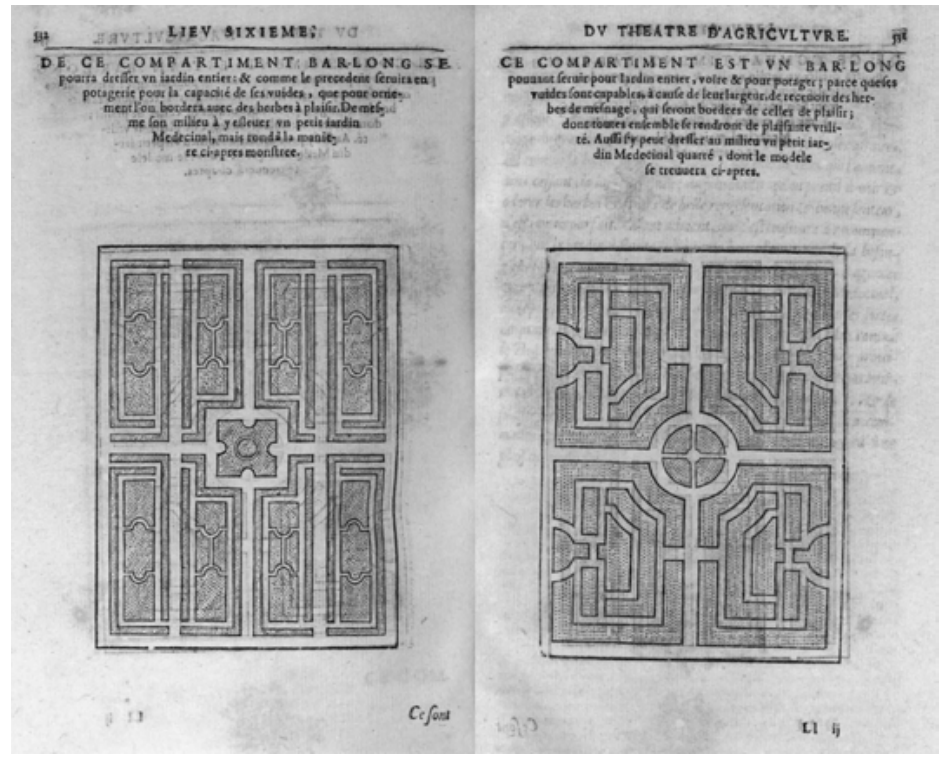

1 Musterentwürfe für schöne und nützliche Gärten von Olivier de Serres, Anfang 17. Jahrhundert.

Baumgarten empfiehlt Serres die Verwendung von Spalierobst, das er für sehr schmückend hält: »Selbst im Winter sind diese Bäume nicht bar der Schönheit, wenn ihr nacktes, kunstvoll verschlungenes Geäst seine große Anmut zeigt« (zit. n. Wimmer 1989, 85).

Joseph Addison schildert 1712 einen imaginären Garten, in dem die verschiedenen funktionalen Gartenräume und auch die Pflanzenarten in ihrer Anordnung »ein Gemisch « bilden. Sein Küchengarten ist in seinen Augen »immer ein reizender Anblick gewesen « und es macht ihm »mehr Vergnügen, die langen Reihen meiner Kohl- und Krautköpfe zu betrachten, die alle in ihrem vollen natürlichen Grün und Wohlgeruch aufschießen, als die zarten Pflanzen fremder Länder durch künstliche Hitze mühsam am Leben erhalten zu sehen« (zit. n. Wimmer 1989, 152).

Auch in den großen, ehemals herrschaftlichen Parkanlagen gehören diese Aspekte zusammen: Der Obstgarten, der Gemüsegarten und die Treibhäuser waren bis ins 20. Jahrhundert wichtige Bestandteile der Parks. Seit aus diesen Gärten aber kein Hofstaat mehr versorgt werden musste und seit es auch für die Gärtnerfamilie günstiger war, die Früchte auf dem Markt oder später im Supermarkt zu kaufen, wurden die Nutzgärten in den Parks fast überall aufgegeben. Als in den 1970er und 80er Jahren gartendenkmalpflegerisches Handeln in Deutschland begann, waren Obst- und Gemüsegärten in den Parkanlagen nicht mehr existent. Und der zunächst stark kunsthistorisch geprägte Blick auf die Parkanlagen als Gartenkunstwerke vermisste sie auch nicht. Diese Einstellung hat sich in den letzten zwei Jahrzehnten zu verändern begonnen. 


\section{Heutige Situation}

Die Kombination von Nützlichem und Zierendem belegt eine Ganzheitlichkeit in der Gartenkultur, die in der heutigen Zeit beim Publikum wieder nachgefragt ist und honoriert wird. Eine Anlage, die als Vorreiter dieser Bewegung angesehen werden kann, ist der Hofgarten Veitshöchheim in der Verantwortung der Bayerischen Schlösserverwaltung. Dieser Garten, der seine Blütezeit zwischen 1755 und 1779 erlebte, war vom frühen 18. bis ins 20. Jahrhundert durch eine innige Verzahnung von Zier- und Nutzpflanzenkulturen geprägt (Albert/ Ehberger 2006). Von 12 ha Gesamtfläche entfiel allein 1 ha auf den Küchengarten, der beiderseits des Hauptzuganges zum Schloss gelegen war. Umfangreiche Nutzpflanzenkulturen gab es aber auch in den großen Boskettzonen, wo innerhalb der Heckenstrukturen überwiegend kleinkronige Obstbäume gepflanzt waren. 1997 begann man hier mit der Wiederanlage des Küchengartens und der Wiederanpflanzung von sogenanntem Franzobst in den Boskettquartieren. »Das ausschlaggebende Argument für die Wiederbelebung des Nutzpflanzenanbaus war die Erkenntnis, dass den Besuchern nur auf diese Weise ein annähernd authentisches Bild des ursprünglichen Gartenkunstwerks zu vermitteln sei und ehemalige Funktionszusammenhänge sichtbar gemacht werden können « - so formuliert es Jost Albert auf der Homepage des Küchengartennetzwerks (Albert o. J.). Dieser Verein, der vor etwa 20 Jahren wesentlich von ihm mit initiiert wurde, ist ein Zusammenschluss von Fachleuten, die sich für die Wiederbelebung von Nutzpflanzenkulturen in historischen Gärten einsetzen.

Neben der Bayerischen Schlösserverwaltung, die ab 1998 auch den Küchengarten im Würzburger Hofgarten wieder anlegte, ist die Gartenverwaltung der Staatlichen Schlösser und Gärten Hessen aktiv: Hier stellte man sowohl den Obstgarten im Schlosspark Bad Homburg als auch die Nutzgartenpartien im Prinz-Georg-Garten in Darmstadt wieder her. In diesem, in seinen Ursprüngen in das frühe 17. Jahrhundert zurückreichenden Garten wurden nachweislich ab dem 18. Jahrhundert bis Anfang des 20. Jahrhunderts große Bereiche als Gemüse- und Obstgarten genutzt. Zwischen 1998 und 2005 wurde die Gartenanlage restauriert, mit dem Ziel, »den besonderen Charakter und die Eigenart des historischen Gartens wieder deutlich hervorzuheben«. Besonderen Wert legte man dabei auf die historische Pflanzenverwendung "mit ihrer charakteristischen Kombination vielfältiger Zierund Nutzpflanzenarten, der Kräuter und [...] den Kübelpflanzen« (Fischer/Handke o. J.). Der Küchengarten der Eutiner Residenz in Schleswig-Holstein wurde zur Landesgartenschau 2016 wiederhergestellt. Dieser 1,8 ha große Küchengarten, der um 1790 in die neue landschaftliche Gestaltung der Anlage integriert wurde, war nach englischem Vorbild des Walled garden mit einer Backsteinmauer umgeben. Nach einem Wettbewerb zur »Revitalisierung « im Jahr 2005 wurde ab 2007 mit Instandsetzungsarbeiten an den erhaltenen baulichen Elementen begonnen, seit 2015 erfolgt eine Kultivierung von Zwergobst. »Langfristiges Ziel ist hierbei, den Garten nach ökologischen Gesichtspunkten zu bewirtschaften und vergessene traditionelle gärtnerische Techniken einer breiten Öffentlichkeit zu vermitteln. Die Arbeiten werden durch regelmäßig stattfindende Parkpflegeseminare mit der Eutiner Bevölkerung und eingeladenen Fachleuten unterstützt« (Ickrath o. J.). 


\section{Eigenproduktion von Gehölzen als wichtigstes Baumaterial Historische Situation in den Parkanlagen des 18. und 19. Jahrhunderts}

Gehölze für die Anlage von Gärten und Parks wurden bis in die Mitte des 18. Jahrhunderts überwiegend aus dem Wald oder von Privatleuten in der näheren Umgebung beschafft. Zu großen Teilen bestanden die Gärten bis in diese Zeit somit aus gebietseigenem, autochthonem Pflanzmaterial. Damit waren sie an die Klima- und meist auch die Bodenverhältnisse des Ortes angepasst, was im Hinblick auf ihr Gedeihen positiv zu werten ist. Auf der anderen Seite waren die Bedingungen für ein erfolgreiches Anwachsen am neuen Standort aber häufig dadurch erschwert, dass - zuweilen auch schon relativ große - Gehölze erstmals umgepflanzt wurden und daher Schäden am Wurzelwerk zu häufig beklagten Ausfällen führten (Butenschön 2014, 210).

Mit dem Aufkommen des Landschaftsgartens auf dem Kontinent und der Verwendung amerikanischer Gehölzarten ab den 1760er Jahren begann die Anlage von Park-Baumschulen bei hochadeligen Lustgärten, wie Schwetzingen, Wörlitz, Weimar u.a. In Hannover-Herrenhausen wurde im Jahr 1767 auf Anordnung des in London residierenden Landesherren Georg III. eine als Plantage bezeichnete Baumschule angelegt. Hier sollten ausländische Gehölze auf ihre Klimaverträglichkeit im Hannoverschen getestet und vermehrt werden (Palm 2012). Einen ähnlichen Ansatz verfolgte man in Kassel-Wilhelmshöhe, wo dem neu eingestellten Hofgärtner Schwarzkopf 1766 zur Aufgabe gestellt wurde, für heimische, aber auch »fremde« Parkbäume Anzuchtquartiere und Baumschulen anzulegen, um dauerhaft Pflanzmaterial für die Unterhaltung und den Ausbau des Parks zur Verfügung stellen zu können. Gleiches forderte man 1787 in Potsdam von Heinrich Ludwig Manger, als er Inspektor der Gartenverwaltung wurde. Seine Dienstanweisung enthielt den expliziten Hinweis auf die Anlage von Revier-Baumschulen, damit daraus "die alten abgestorbenen und andere ausgegangene Bäume, sowohl in den Gärten, Alleen und Plantagen, als in den Treibehäusern zu allen Zeiten [...] recrutiret werden können «' . Diese Baumschulen sollten also der kontinuierlichen Versorgung der Parks mit Gehölzen für Nachpflanzungen dienen. Die Notwendigkeit dafür ergab sich regelmäßig auf Grund der Witterungsverhältnisse, z.B. durch Ausfälle in Folge des sehr kalten Winters 1788/89. Johann Gottlob Schulze, der drei Jahre später das Amt des Garteninspektors übernahm, sah die einzelnen kleinen Baumschulen als nicht mehr ausreichend an und legte in den Jahren 1790 bis 1795 eine etwa 4 ha große, mustergültige Baumschule am Südrand des Parks von Sanssouci an.

Für die Neugestaltung des Tiergartens in Berlin standen Peter Joseph Lenné 1833 dann schon mehrere Baumschulen zur Verfügung. Neben der Landesbaumschule in Potsdam waren dies die in den 1790er Jahren entstandene Baumschule innerhalb des Tiergartens (zwischen Bellevue-Allee und damaliger Charlottenburger Chaussee, heute Straße des

1 Niedersächsisches Landesarchiv - Staatsarchiv Oldenburg, Bestand 271-25, Nr. 52, Nachlass Karoline Schulze, 67 (3A). 
17. Juni) sowie die Baumschulen in Moabit, Tegel und Schönhausen. Darüber hinaus wurden beim Auslichten der Bestände Junggehölze zur Neupflanzung geborgen, eingeschlagen oder sofort an neue Standorte verpflanzt (Wendland 1993, 101).

Friedrich Ludwig von Sckell widmete in seinem Werk Beiträge zur bildenden Gartenkunst der Anlage und dem Betrieb von Baumschulen ein eigenes Kapitel. Er empfahl allen, die einen Park anlegen wollen, »mit der Anlage einer Baumschule den Anfang zu machen « (Sckell 1825, 231). Sckells zentrales Argument war folgendes: »Pflanzen, in loco gezogen, sind schon da, wo sie die Gärten der Natur schmücken sollen, sowohl an das Klima, wie an die Erde gewöhnt, so daß man auf ihr Gedeihen und Fortkommen weit sicherer rechnen kann, als auf solche Pflanzen, die man erst aus entfernten Gegenden und Himmelsstrichen muss kommen lassen« (ebd.). Parkbaumschulen wurden so seit dem 18. Jahrhundert zum integralen Bestandteil großer Parkanlagen.

\section{Heutige Situation}

Gegen Ende des 19. Jahrhunderts wurde dieses Prinzip aufgegeben, da sich das Pflanzmaterial oftmals preiswerter in großen Handelsbaumschulen zukaufen ließ. Ein reichhaltiges Angebot und gesunkene Transportkosten ließen es zweckmäßig erscheinen, Gehölze anzukaufen, statt sie selbst zu produzieren. Damit wurde ein Arbeitsschritt in der kontinuierlichen Erhaltung von Gärten und Parks ausgelagert. Diese Arbeitsteilung bietet aber nicht nur Vorteile für die Gehölze und die Parks als Gartendenkmale. Ihre pflanzliche Substanz, die im Laufe der Zeit zu historischer Substanz im denkmalpflegerischen Sinne heranwächst, wird durch dieses Vorgehen vereinheitlicht und verflacht. Man hat auf die vielfältigen Vorteile einer Eigenproduktion verzichtet, die erst in letzter Zeit wieder stärker wahrgenommen werden. Zum einen wachsen die Gehölze in der Umgebung und mit den abiotischen und biotischen Bedingungen auf, unter denen sie leben sollen - dieses Argument nannte Anfang des 19. Jahrhunderts bereits Sckell. Man kann also an den spezifischen Standort gut angepasste Exemplare produzieren. Zudem sind keine Transportschäden zu befürchten und der Prozess des Umpflanzens erfolgt voraussichtlich schonender, da er in eigener Verantwortung geschieht. Außerdem erhält man durch Vermehrung von im Park vorhandenen Arten und Sorten die genetische Vielfalt der Gehölze. Dieser Genpool bewahrt Optionen auch für Züchtung und Weiterentwicklung.

Heute ist es daher vor allem eine denkmalpflegerische Frage des Erhalts von authentischem Pflanzenmaterial, die Gartendenkmalpfleger dazu bewegt, sich um eine eigene Gehölznachzucht zu bemühen. Im Pückler-Park Branitz hat man dafür auf dem Gärtnereigelände ein Baumschulquartier eingerichtet (Wecke 2014, 153-158), in dem beispielsweise eine Grau-Pappel (Populus $\times$ canescens) genetisch identisch vermehrt wird, die von Pückler am Heiligen Berg im Branitzer Park gepflanzt wurde und die sich durch ihren markanten trauernden Wuchs mit überhängenden Ästen auszeichnet (Abb. 2). Solche besonderen Sorten oder Varietäten, die gar nicht unbedingt namentlich bekannt sein müssen, aber für die 


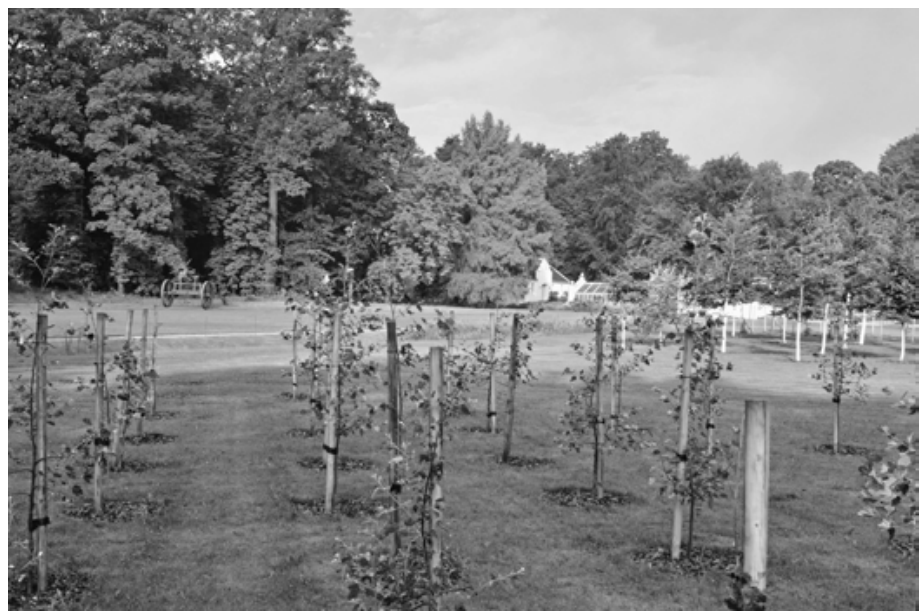

2 Nachzucht einer Grau-Pappel in der sog. Baumuniversität des Pückler-Parks Branitz, 2013.

Parkanlagen prägend sein können, sind natürlich am besten vor Ort und in eigener Produktion zu erhalten.

Darüber hinaus kann eine Park-Baumschule aber auch dazu beitragen, die Sortenvielfalt innerhalb einer historischen Parkanlage zu erhalten. Das historische Arboretum im Park von Bad Muskau ist nur ein Beispiel dafür, dass sich durch regionale Produktion die genetische Vielfalt innerhalb einer Art erhöht hat (Richter 2014, 185f.). Dort sind durch Züchtung oder Selektion sogar neue Sorten entstanden, wie beispielsweise Quercus petraea >Muscaviense «. Derartige historische Gehölzsorten sind im Sortiment moderner Baumschulen kaum verfügbar, da ihre Produktion aus betriebswirtschaftlicher Sicht unrentabel ist. Um sie zu bewahren, muss also eine Nachzucht auf der Basis überkommener Altgehölze in den entsprechenden Parkanlagen stattfinden. Zudem ist natürlich denkbar, dass auch künftig in Park-Baumschulen neue Sorten entstehen können. Damit würden solche Baumschulen einen Beitrag zur Förderung der Biodiversität auf Gen-Ebene leisten.

\section{Pflege und Management in geschlossenen Stoffkreisläufen Historische Situation in den Parkanlagen des 18. und 19. Jahrhunderts}

Bis zum Ende des 19. Jahrhunderts war es eine Selbstverständlichkeit, bei der Bewirtschaftung eines Gartens oder Parks alle Produkte und Erzeugnisse dieser Flächen möglichst sinnvoll zu nutzen. Das betraf nicht nur pflanzliche Biomasse wie Gras, Laub oder Holz, sondern auch tierische Produkte, denn die Haltung von Federvieh, Schweinen, Schafen, Rindern und Pferden war für die Gärtner ebenso üblich. Für diese Tiere konnte der pflanzli- 


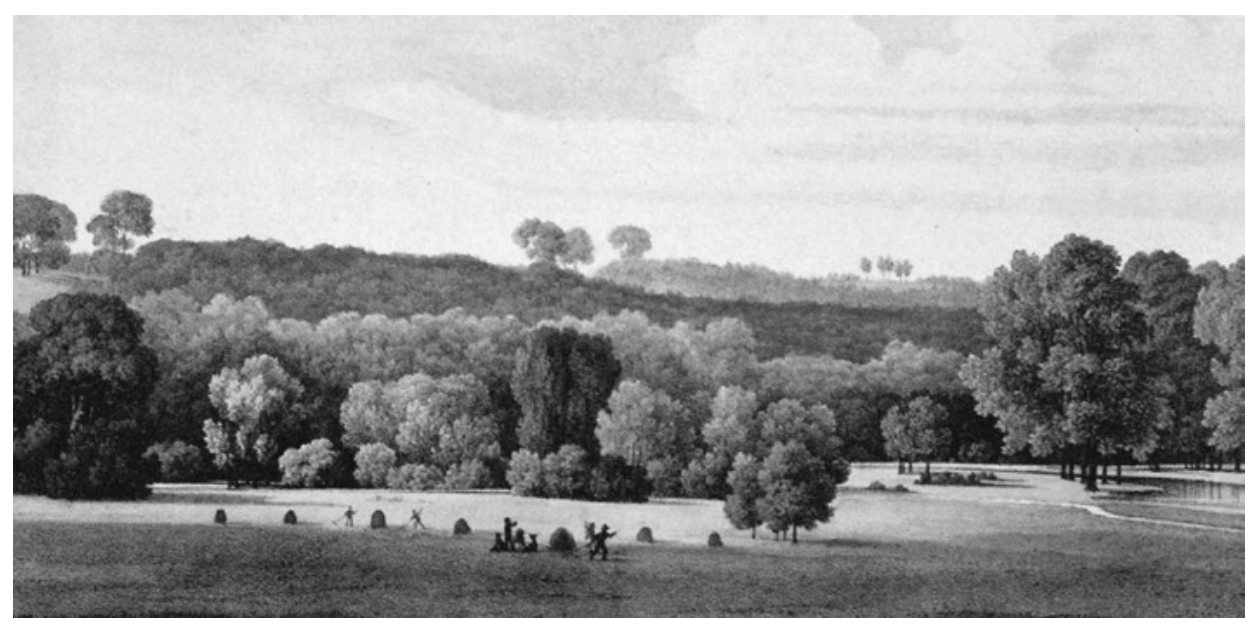

3 Blick von der Gloriette über die Seewiese im Muskauer Park. Kolorierte Lithographie von O. Hermann nach einem Aquarell von A. W. Schirmer. In: Pückler-Muskau, Hermann Fürst von (1834): Andeutungen über Landschaftsgärtnerei. Stuttgart, Tafel XVIII.

che Aufwuchs des Parks verwendet werden, indem auf Parkwiesen Heu produziert (Abb. 3) und als Winterfutter genutzt wurde oder die Tiere direkt im Park weideten. Ihre Ausscheidungen düngten so direkt die Flächen oder man verwendete sie zur Produktion eines guten Düngers. In Krünitz' Oekonomischer Encyklopädie heißt es: »Der Dünger wird entweder aus dem mineralischen und Erdenreiche selbst, oder aus dem vegetabilischen und Pflanzenreiche, oder aus dem animalischen und Thierreiche genommen; jedoch gebührt dem Miste, der aus dem thierischen und Pflanzenreiche entstehet, vor allen andern der [sic] Vorzug« (Krünitz 1776, Bd. 9, 690).

Schon im 18. Jahrhundert wussten die Fachleute, welche Bodenverbesserungsmaßnahmen für die Bewirtschaftung von Gärten und Parks notwendig und sinnvoll waren und wie guter Dünger als Verrottungsprodukt von biologischen Abfallstoffen zu erzeugen war. Auch bei der Anlage von Parks wurde darauf geachtet, wie am Beispiel der Umgestaltung des Großen Tiergartens in Berlin von Peter Joseph Lenné gezeigt werden kann. So empfahl Lenné 1832 in seiner Denkschrift zum Entwurf Bodenverbesserungsmaßnahmen für nährstoffarme Partien, auf denen Rasenflächen entstehen sollten: »Die niedrige Lage des Terrains ist der Rasenbildung günstig, und wo dasselbe sandig und trocken ist, kann man ihm mit der Rasenerde der Wiesen und Moorgründe zu Hilfe kommen. $\ll^{2}$ Er wusste auch, dass solche Flächen, die keine natürlichen Wiesenstandorte darstellten, eine kontinuierliche Pflege benötigten, was er in seiner Denkschrift über die Unterhaltung des Tiergartens vom 1.1.1839 darlegte: 


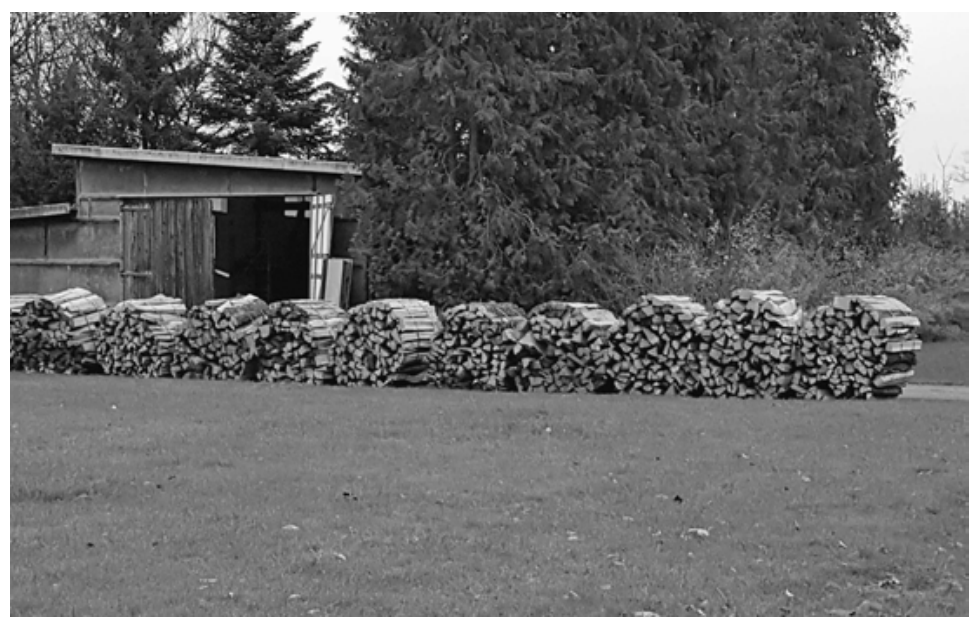

4 Brennholz-Lager auf dem Betriebshof einer historischen Parkanlage, 2017.

Die Rasenplätze, die den Hauptschmuck der Anlagen begründen, sind mit geringer Ausnahme keine natürlichen Wiesen, der größte Teil ist auf sandigem Höheboden und im Schatten dichter Wald- und Hainmassen künstlich erzeugt. Diese können daher auch nur durch sorgfältige Pflege künstlich in ihrem Schmuck gehalten werden; neue Besamungen sind daher von Zeit zu Zeit erforderlich, die vorhandene Grasnarbe muß durch Übertragung geeigneter Erdarten und Dungstoffe genährt und erfrischt [Hervorhebung SB] werden (zit. n. Hinz 1937, 165f.).

Auch die vorgesehenen Blumenbeete müssten nach Einschätzung Lennés noch gedüngt werden, da der Boden noch der »erforderlichen nährenden Substanzen« (Hinz 1937, 166) entbehre. Das Einbringen von Nährstoffen auf Flächen, die einen hohen Bedarf an solchen Substanzen haben, war für die Gärtner eine in der Ausbildung erlernte übliche Praxis. Vor der Erfindung des Mineraldüngers benutzte man als Ausgangsmaterial im Wesentlichen die Substanzen, die im Park und in der Haushaltung anfielen.

\section{Heutige Situation}

Ebenso wie die Produktion von Bäumen und Sträuchern wurden auch andere Arbeiten, die zur Parkpflege und Unterhaltung nötig sind, im 20. Jahrhundert im Zuge einer immer stärkeren Arbeitsteilung, die in vielen Bereichen in der Gesellschaft stattfand, aus den Pflegebetrieben ausgelagert. So wird heute in Parks und Gärten entstehende Biomasse in vielen Fällen auf verschiedenen Wegen aus den Anlagen ausgeführt. Beispielsweise verkaufen viele Parkverwaltungen das anfallende Holz als Brennholz an Privatkunden, wobei dieser Verkauf in aller Regel unter Wert erfolgt. Man ist froh, das Material los zu werden und investiert dafür sogar in eine Professionalisierung der Vermarktung, z.B. für den Verkauf von Brennholz, das gebündelt für die Abnehmer vorgehalten wird (Abb. 4). 
Außerdem erfolgt eine Separierung der unterschiedlichen Grünabfälle. Manche Parkverwaltungen lagern Laub getrennt und lassen es anrotten, um es dann an Landwirte abzugeben, die diesen Rohkompost als Düngematerial auf Ackerflächen ausbringen. In der Regel erfolgt zwar in den Gärten und Parks eine Kompostwirtschaft, also eine mehr oder weniger geordnete Verrottung des anfallenden Grünguts zu Komposterde. Häufig wird diese Erde aber gar nicht benutzt und lagert für lange Zeit auf dem Betriebshof, begrünt dann, ist damit von Unkrautsamen durchsetzt und verliert ihre Qualität von Jahr zu Jahr. Neben diesen Beispielen, die zumindest noch ein wenig Geld einbringen oder jedenfalls keine Kosten verursachen, kommt es auch häufig vor, dass man Biomasse als Grünabfall kostenpflichtig entsorgen lässt. Insgesamt werden mit der Biomasse auch Nährstoffe aus dem natürlichen Stoffkreislauf des Gartens entzogen, die über den Zukauf von Düngern und Spezialerden wieder ins System geholt werden (müssen) - da sich andernfalls langfristig die Standortqualitäten verschlechtern.

\section{Handlungsempfehlungen für die Praxis}

Gärten und Parks mit einem ganzheitlichen Ansatz zu pflegen und zu bewirtschaften, ist generell ein sinnvolles gartendenkmalpflegerisches Handeln. Dazu gehören auch die Integration von Nutzpflanzen, das Betreiben von Parkbaumschulen und das Streben nach geschlossenen Stoffkreisläufen in der Parkpflege als zentrale Aspekte von Nachhaltigkeit.

- Wiederanlage ehemaliger Nutzpflanzenquartiere oder Küchengärten

Anlage und Betrieb von Nutzgärten durch Parkverwaltungen zeigen den Besuchern ein authentisches ganzheitliches System des Gartens oder Parks. Solche Quartiere übernehmen in historischen Gärten auch eine Vorbildfunktion für die Besucher und geben Anregungen zum Eigenanbau - in einer Zeit, in der die lokale Produktion von Lebensmitteln eine Antwort (unter vielen notwendigen) auf den Klimawandel ist. Das dort erzeugte Obst und Gemüse sollte möglichst lokal genutzt werden. So könnten beispielsweise Bohnen oder Salat in Parkrestaurants verarbeitet werden. Ebenso könnten parkeigene Äpfel historischer Sorten an einem Kiosk zum Verkauf angeboten werden. Die Bewirtschaftung sollte bestenfalls mit eigenen Gärtnerinnen und Gärtnern erfolgen, wobei damit zu rechnen ist, dass auch für nicht besonders große Nutzgärten eine zusätzliche »Küchengärtner-Stelle« zu besetzen ist.

- Anlage von Baumschulquartieren für die Nachzucht parkeigener Gehölze

Der Betrieb von Parkbaumschulen ermöglicht die Erzeugung und das Vorhalten von Nachkommen alter Parkgehölze, um das genetische Material, aus dem das Gartendenkmal aufgebaut ist, authentisch zu erhalten. Dafür können gekeimte Samen ebenso dienen wie Reiser, Absenker oder andere Formen der vegetativen Vermehrung. Eine geregelte Baumschulwirtschaft im Park erhält die genetische Vielfalt und mit ihr die spezifische Eigenart des Parks. 


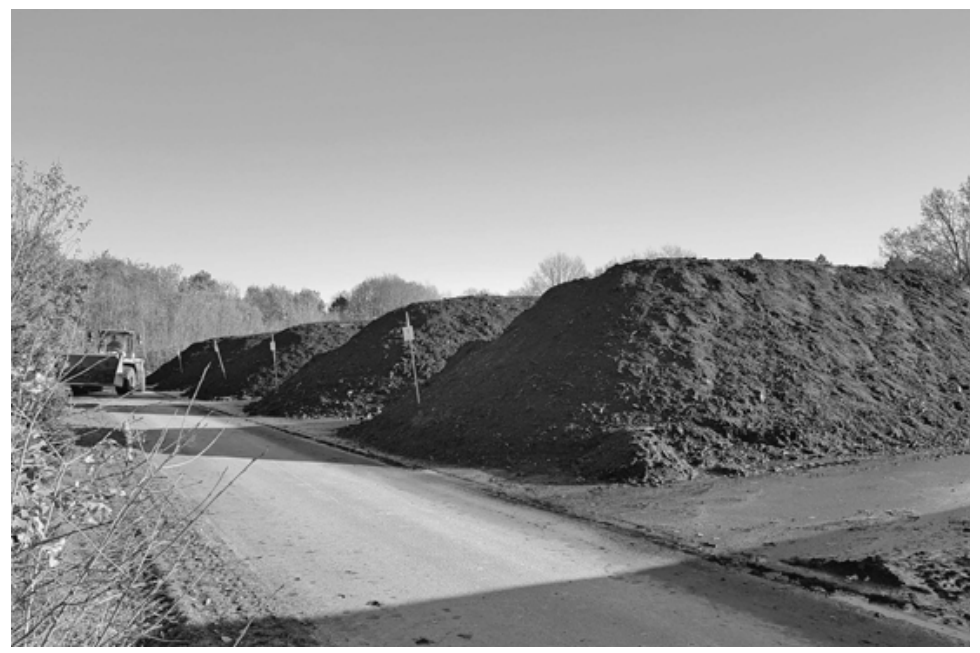

5 Vorbildlicher Kompostplatz zur Produktion von Komposterde aus der im eigenen Betrieb anfallenden Biomasse auf dem Hauptfriedhof Ohlsdorf, Hamburg.

\section{- Parkpflege mit eigenem Betriebshof}

Die Pflege historischer Parks sollte mit einem eigenen Betriebshof erfolgen, auf dem das Konzept »no waste« verfolgt wird. Im Park entstehende Biomasse darf nicht als »Grünabfall« angesehen werden, sondern ist als wertvolles »Grüngut« zu betrachten, das bewirtschaftet werden kann. Möglichkeiten der direkten Nutzung vor Ort bieten das Mulchen und die Beweidung von Grünlandflächen. Damit bleiben das Material oder zumindest die Nährstoffe in umgewandelter Form direkt vor Ort, wodurch die Qualität des Bodens erhalten wird. Auf dem Gelände und im System bleiben die Nährstoffe auch durch eine geregelte Kompostwirtschaft (Abb. 5). Bei entsprechender Bewirtschaftung kann in jedem historischen Garten eine hochwertige Komposterde erzeugt werden, die frei von Unkrautsamen ist, da die notwendigen Temperaturen in der Rotte erreicht werden. Eine Optimierung des Komposts lässt sich durch eine Karbonisierung von überschüssiger Biomasse (beispielsweise Holz oder Laub) erreichen - ein Prozess, bei dem biologisches Material unter hohen Temperaturen zu Pflanzenkohle verarbeitet wird (Terytze/Wagner 2015). Ein Zusatz solcher Pflanzenkohle zum Kompost verbessert das Substrat enorm und lässt es zum Langzeitdünger werden. Eine weitere Verarbeitung zu einem Torfsubstitut ist möglich.

- Hervorhebung und Bewerbung des ganzheitlichen Ansatzes im Marketing-Konzept Die Tatsache, dass zur Erhaltung historischer Gärten eine qualifizierte und nachhaltige Parkpflege gehört, sollte nicht `hinter den Kulissen` verschwinden. Die Arbeit der Gärtnerinnen und Gärtner darf als Bestandteil des Kulturerbes ebenso präsentiert werden wie das von ihnen gepflegte Bild des Parks. 


\section{Archivalien}

Geheimes Staatsarchiv Preußischer Kulturbesitz (GStA PK) Rep. 151 IC.

Niedersächsisches Landesarchiv - Staatsarchiv Oldenburg, Bestand 271-25, Nr. 52, Nachlass Karoline Schulze, $67(3 \mathrm{~A})$.

\section{Literaturverzeichnis}

Albert, Jost (o. J.): Der Küchengarten im Veitshöchheimer Hofgarten. http://www.kuechengarten.net/index.php ?content $=05$ gaerten $\&$ sub=Veitshoechheim (26.04.2018).

Albert, Jost; Ehberger, Gabriele (2006): "Es kommen immer Leit aus Würzburg und Frembde hierher" - Zur Geschichte des Rokokogartens Veitshöchheim. Begleitheft zur Ausstellung. München: Bayerische Schlösserverwaltung.

Butenschön, Sylvia (2014): Parkbaumschulen - Zentren regional angepasster Gehölzvielfalt in Vergangenheit und Zukunft. In: Historische Gärten im Klimawandel. Empfehlungen zur Bewahrung. Hg. von der Generaldirektion der Stiftung Preußische Schlösser und Gärten Berlin-Brandenburg. Leipzig: Edition Leipzig, 210-213.

Fischer, Christine; Handke, Manfred (o. J.): Der Prinz-Georg-Garten in Darmstadt. http://www.kuechengarten. net/index.php?content=05gaerten\&sub=Darmstadt (26.04.2018)

Hinz, Gerhard (1937): Peter Josef Lenné und seine bedeutendsten Schöpfungen in Berlin und Potsdam. Berlin: Deutscher Kunstverlag.

Ickrath, Anja (o.J.): Der Küchengarten der Eutiner Residenz. http://www.kuechengarten.net/index.php?content= 05gaerten\&sub=Eutin (26.04.2018).

Krünitz, Johann Georg (1776): Oekonomische Encyklopädie oder allgemeines System der Staats- Stadt- Hausund Landwirthschaft, Bd. 9. Berlin: Pauli.

Palm, Heike (2012): Die landesherrliche Plantage in Herrenhausen. Ein Instrument zur Förderung des Obstbaus und der Gartenkultur im Kurfürstentum Hannover. In: Frühe Baumschulen in Deutschland. Zum Nutzen, zur Zierde und zum Besten des Landes. Hg. von Sylvia Butenschön. Berlin: Universitätsverlag TU Berlin, 69-109.

Pückler-Muskau, Hermann Fürst von (1834/1977): Andeutungen über Landschaftsgärtnerei. Nachdruck von 1977. Stuttgart: DVA.

Richter, Susanne (2014): Gehölzvielfalt in den Gärten von Eduard Petzold. Ansätze zur Revitalsierung in Theorie und Praxis. In: Wiedergeburt von Baumgiganten. Vermehrung wertvoller Gehölze als Instrument der Gartendenkmalpflege und des Naturschutzes. Hg. von Claudius Wecke und Karola Weber im Auftrag der Stiftung Fürst-Pückler-Museum Park und Schloss Branitz. Berlin: be.bra, 183-196.

Sckell, Friedrich Ludwig von (1825): Beitraege zur bildenden Gartenkunst für angehende Gartenkünstler und Gartenliebhaber. München: Lindauer.

Serres, Olivier de (1651): Le théatre d'Agriculture. Genf: Chouet.

Terytze, Konstantin; Wagner, Robert (2015): Endbericht zum Forschungsvorhaben »Schließung von Kreisläufen durch Energie- und Stoffstrommanagement bei Nutzung der Terra-Preta-Technologie im Botanischen Garten im Hinblick auf Ressourceneffizienz und Klimaschutz - Modellprojekt Urban farming“ (TerraBoGa). Berlin: Freie Universität. https://refubium.fu-berlin.de/handle/fub188/15068 (25.09.2018).

Wecke, Claudius (2014): Die Baumuniversität im Fürst-Pückler-Park Branitz. In: Wiedergeburt von Baumgiganten. Vermehrung wertvoller Gehölze als Instrument der Gartendenkmalpflege und des Naturschutzes. Hg. von Claudius Wecke und Karola Weber im Auftrag der Stiftung Fürst-Pückler-Museum Park und Schloss Branitz. Berlin: be.bra, 145-162.

Wendland, Folkwin (1993): Der große Tiergarten in Berlin. Seine Geschichte und Entwicklung in fünf Jahrhunderten. Berlin: Gebr. Mann.

Wimmer, Clemens Alexander (1989): Geschichte der Gartentheorie. Darmstadt: Wissenschaftliche Buchgesellschaft. 


\section{Bildnachweis}

1 Bayerische StaatsBibliothek digital, 4 Oecon. 327, Bl. 532, http://mdz-nbn-resolving.de/urn:nbn:de:bvb:12bsb10229141-3; 2 Stiftung Fürst-Pückler-Museum Park und Schloss Branitz, Karola Weber; 3 Original: Stiftung »Fürst-Pückler-Park Bad Muskau«; 4, 5 Sylvia Butenschön. 